\title{
On IO-copying and mildly-context sensitive formalisms
}

\author{
Pierre Bourreau ${ }^{1}$, Laura Kallmeyer ${ }^{2}$, and Sylvain Salvati ${ }^{1}$ \\ 1 Université Bordeaux 1 \\ 351, Cours de la Libération \\ 33405 Talence Cedex, France \\ \{bourreau, salvati\}@labri.fr \\ 2 Heine-Heinrich Universitat Dusseldorf \\ Universitatstr. 1 \\ 40225 Dusseldorf, Germany \\ kallmeyer@phil.uni-duesseldorf.de
}

\begin{abstract}
The class of mildly context-sensitive languages is commonly accepted as fulfilling the requirements to describe natural language. Many formalisms are known to generate languages which belong to this class, such as tree-adjoining grammars, multiple context-free grammars or abstract categorial grammars. All these formalisms share the property of being describe thanks to linear transformations, i.e. which avoid erasing or copying of material along derivations. We show that restricted copying operations allow defining mildly context-sensitive languages, thanks to the introduction of a new operation: IO-substition of languages.
\end{abstract}

Keywords: mildly context-sensitive languages, abstract families of languages, IO substitution, PMCFG.

\section{Introduction}

The question of the amount of expressive power needed in order to deal with natural languages is still an open question. In this context, the notion of mild-context-sensitivity has been proposed. A grammar formalism is mildly context-sensitive if a) it is more powerful that $\mathrm{CFG}, \mathrm{b}$ ) it generates only languages of constant growth, c) it can generate languages that describe a limited amount of cross-serial dependencies and d) it is polynomially parsable. A well-known class of formalisms that is still mildly contextsensitive is the class containing Linear Context-Free Rewriting Systems (LCFRS) and equivalent formalisms such as Multiple Context-Free Grammars (MCFG), Minimalist Grammar (MG) and set-local multicomponent Tree Adjoining Grammars (MCTAG).

This paper explores ways to characterize formally and linguistically interesting extensions of the class of LCFRL/MFCL that are still mildly context-sensitive. It follows ideas from [Kal10] where a mildly context-sensitive LCFRS extension is defined that allows for a limited amount of copying during derivations. The definition of this formalisms called Literal Movement Grammars of constant non-linearity (CNL-LMG) is, however, based on properties of possible derivations in a grammar. Furthermore, it lacks an independent characterization of the resulting class of string languages. 
In this paper, we define first an operation on string languages that amounts to copying a substring into different places, called IO-substitution. The idea is roughly that we generate some string $w_{2}$ and a string $w_{1}$ that contains several occurrences of a variable $x$. This variable marks all the positions where the string $w_{2}$ gets copied to. An important point is that the string that gets copied does not increase while being copied. Once we have such a copying operation, we define the closure of MCFL/LCFRL under this operation as a new class IO-MCFL. We show that the languages in this class still have the constant-growth property.

As an example, consider the languages $(x d)^{*} x$ and $a^{*}$. IO-substitution of $x$ in the first language by all possible words from the second language means fixing a word $a^{n}$ from the second and replacing all occurrences of $x$ in words from the first by $a^{n}$. As a result, we obtain $\left\{\left(a^{n} d\right)^{m} a^{n} \mid n, m \geq 0\right\}$ which is a generalization of the counting language that is not a MCFL [Kal10].

In parallel to the definition of IO-substitution on strings, we then characterize possible rules in Literal Movement Grammars (LGM), an MCFG-extension, that describe exactly the IO-subsitition operation. Based on this, a new grammar formalism is defined that turns out to be mildly context-sensitive while being a proper extension of MCFG.

The structure of the paper is as follows: ... to be written ...

\section{Preliminaries}

\subsection{Mildly context-sensitive languages}

Let us consider a countable set $\Sigma$, called an alphabet. We write $\Sigma^{*}=(\Sigma, \cdot, \epsilon)$ the (free) monoid on $\Sigma$, where - is the operation of concatenation, and $\epsilon$ the identity element. Any set $L \subseteq \Sigma^{*}$ is called a language on $\Sigma$.

Given a language $L \in \Sigma^{*}$, and $w$ one of its elements (called words), we define the usual notions of length and number of occurrences of a letter $a \in \Sigma$ in $w$ by induction on $w:|\epsilon|=0,|w|=1$ if $w \in \Sigma$ and $\left|w_{1} \cdot w_{2}\right|=\left|w_{1}\right|+\left|w_{2}\right|$, for the length of $w$ and $|\epsilon|_{a}=0$, $|w|_{a}=1$ if $w=a,|w|_{a}=0$ if $w \in \Sigma-\{a\}$ and $\left|w_{1} \cdot w_{2}\right|_{a}=\left|w_{1}\right|_{a}+\left|w_{2}\right|_{a}$ for the number of occurrences of $a$ in $w$.

The class MCSL of mildly context-sensitive languages [Jos85,Wei88] is defined as the smallest set such that:

- MCSL contains all context-free languages.

- some restricted crossing-dependencies are taken in account by the languages $L \in$ MCSL

- every language $L \in$ MCSL verifies the constant-growth property.

- every language $L \in$ MCSL is recognizable in polynomial-time.

The first property specifies that MCSL falls between the classes of context-free languages and context-sensitive languages in Chomsky's hierarchy: CFL $\subseteq$ MCSL $\subseteq$ CSL. While vague, the second property ensures this class is bigger than the class of context-free languages. Hence, these two properties are easily ensured when defining some new formalisms. 
This work mainly focuses on the last two properties. For the constant-growth property, we consider the following definition [Kal10], which is stronger than the original definition in [Wei88], and is based on the commonly-known notion of the Parikh image:

Notation 21 Let us consider an alphabet $\Sigma$ and the vector space $\mathbb{N}^{|\Sigma|}$. Given a letter $a \in \Sigma$ and a vector $\vec{v}$ on $\mathbb{N}^{|\Sigma|}$, we note by $\vec{v}[a]$ the scalar of $\vec{v}$ on the coordinate associated to $a$.

Definition 1. Let us consider a word $w$ in a language $L \subseteq \Sigma^{*}$. The Parikh image of $w$, written $\vec{p}(w)$ is such that, for every $a \in \Sigma, \vec{p}(w)[a]=|w|_{a}$. The Parikh image of $L$ is defined as $\vec{p}(L)=\{\vec{p}(w) \mid w \in L\}$.

Definition 2. A language $L \in \Sigma^{*}$ is said to verify the constant-growth property if there exists a constant $c \in \mathbb{N}-\{0\}$ such that, for every word $w \in L$ verifying $|w|>c$, there exists $\vec{x}, \vec{y} \in \mathbb{N}^{|\Sigma|}$ for which:

1. $\vec{p}(w)=\vec{x}+\vec{y}$ and

2. for every $i \geq 1, \vec{x}+i \vec{y} \in \vec{p}(L)$.

The constant-growth property is a property weaker than the condition of semilinearity:

Definition 3. A set $L$ of vectors on a vector space $\mathbb{N}^{k}$ is called a linear set if $L=\left\{\overrightarrow{x_{0}}+\right.$ $\left.k_{1} \overrightarrow{x_{1}}+\ldots+k_{n} \overrightarrow{x_{n}} \mid n \in \mathbb{N}, k_{1}, \ldots, k_{n} \in \mathbb{N}-\{0\}\right\}$.

A semilinear set is a finite union of linear sets.

Definition 4. A language L is said semilinear if $\vec{p}(L)$ is a semilinear set.

Many formalisms were created to generate languages that belong to MCSL, among which are tree-adjoining grammars (TAGs) [JLT75], multiple context-free grammars (MCFGs) [SMMK91], which will be introduced in the next section, or abstract categorial grammars (ACGs) [dG01,Mus01]. It is known that the class of multiple context-free languages (i.e. languages generated by MCFGs), which we will write MCFL, strictly entails the class of tree-adjoining languages (i.e. languages generated by TAGs). Hence, we consider MCFL as the best approximation of MCSL.

An important property of languages which belong to the class MCFL is that they are semilinear. In this paper, we introduce the operation of IO-substitution on languages in the class MCFL of languages; we will see that this operation allow the definition of a new class of languages which are not semilinear, but verify the constant-growth property.

\subsection{Multiple context-free tree languages}

In what follows, we define a multiple context-free language as the yield of a tree language derived by a linear multiple context-free tree language (linear MCFTG). Thanks to this definition, we will then prove that some specific MCFTGs are polynomial. The tree languages derived by Linear MCFTGs are exactly the tree languages derived by abstract categorial grammars[dG01,Mus01] of trees, and we write this class of languages MCFTL. 
In order to define the formalism of MCFTG, let us first define trees and tree contexts. These objects will be defined as particular $\lambda$-terms types with simple types built on the single type $o$ : given an atomic type $o$, we define the set of types on $o$ as the smallest set $\mathscr{T}_{o}$ such that $o \in \mathscr{T}_{o}$ and $(\alpha \rightarrow \beta) \in \mathscr{T}_{o}$ if $\alpha, \beta \in \mathscr{T}_{o}$. Tthe usual convention of parenthesis are taken: a type $\alpha_{1} \rightarrow\left(\alpha_{2} \rightarrow \alpha_{3}\right)$ will be written $\alpha_{1} \rightarrow \alpha_{2} \rightarrow \alpha_{3}$. Given a type $\alpha \in \mathscr{T}_{o}$, we define the order $\operatorname{ord}(\alpha)$ by induction on $\alpha$ as $\operatorname{ord}(o)=1$, and $\operatorname{ord}\left(\alpha_{1} \rightarrow \alpha_{2}\right)=$ $\max \left(1+\operatorname{ord}\left(\alpha_{1}\right), \operatorname{ord}\left(\alpha_{2}\right)\right)$.

Definition 5. An alphabet $\Sigma$ is said typed if to every element $a$ in $\Sigma$ is associated a type of $\mathscr{T}_{o}$, written $\tau(a)$. A typed alphabet is called an $n$-order alphabet (where $n \geq 0$ ) if $\max _{a \in \Sigma}(\operatorname{ord}(\tau(a))) \leq n$.

In what follows, a type $\underbrace{\alpha \rightarrow \ldots \rightarrow \alpha}_{n} \rightarrow \gamma \in \mathscr{T}_{o}$ will be abreviated into $\alpha^{n} \rightarrow \gamma$.

Given a typed alphabet $\Sigma$ and $n>0$, we note by $\Sigma^{n}$ the smaller subset of $\Sigma$ which contains all the elements of $\Sigma$ which associated type is of order less or equal to $n$.

Definition 6. Let us consider an enumerable typed alphabet $\Sigma$ and a type $\alpha \in \mathscr{T}_{0}$. We define $\mathbb{T}^{\alpha}(\Sigma)$ the trees of type $\alpha$ on $\Sigma$ as the smallest set such that:

- $c \in \mathbb{T}^{\alpha}(\Sigma)$ if $c \in \Sigma$ and $\tau(c)=\alpha$

- $\left(t_{1} t_{2}\right) \in \mathbb{T}^{\alpha}(\Sigma)$ if $t_{1} \in \mathbb{T}^{\gamma \rightarrow \alpha}(\Sigma)$ and $t_{2} \in \mathbb{T}^{\gamma}(\Sigma)$.

The set $\mathbb{T}(\Sigma)$ of trees on $\Sigma$ is defined as $\mathbb{T}(\Sigma)=\mathbb{T}^{o}(\Sigma)$. Moreover, if $\Sigma$ is 2-order, we say that $\mathbb{T}(\Sigma)$ is a pure set of trees.

We take the usual conventions for parenthesis so that a tree $\left(t_{1} t_{2}\right) t_{3}$ is written as $t_{1} t_{2} t_{3}$. The general form of a tree in $\mathbb{T}(\Sigma)$ is therefore $c t_{1} \ldots t_{n}$, where $c \in \Sigma, \tau(c)=\alpha_{1} \rightarrow$ $\ldots \rightarrow \alpha_{n} \rightarrow o$ and for every $1 \leq i \leq n, t_{i} \in \mathbb{T}^{\alpha_{i}}(\Sigma)$.

Given a type $\alpha \in \mathscr{T}_{o}$ and a tree $t \in \mathbb{T}^{\alpha}(\Sigma)$ and $a \in \Sigma$, we define $|t|_{a}$ as the number of occurrences of $a$ in $t$ by induction on $t:|a|_{a}=1,|c|_{a}=0$ if $c \in \Sigma-\{a\}$, and $\left|t_{1} t_{2}\right|_{a}=\left|t_{1}\right|_{a}\left|t_{2}\right|_{a}$.

The yield function is a function which takes a tree $t$ as its inputs and returns the sequence of leafs of $t$ from left to right. Formally, given a typed alphabet $\Sigma$, we define yield: $\mathbb{T}(\Sigma) \mapsto \Sigma^{*}$, as yield $(c)=c$ if $c \in \Sigma$, yield $\left(t_{1} t_{2}\right)=\operatorname{yield}\left(t_{2}\right)$ if $t_{1}=c \in \Sigma$ and yield $\left(t_{1} t_{2}\right)=\operatorname{yield}\left(t_{1}\right) \cdot \operatorname{yield}\left(t_{2}\right)$ otherwise. We extend this notion to sets of trees: given a set of trees $L, y L=\{$ yield $(t) \mid t \in L\}$.

Definition 7. Let us consider a finite second-order alphabet $\Sigma$ and a type $\alpha \in \mathscr{T}_{0}$. We define the set $\mathbb{T}_{[]}^{\alpha}(\Sigma)$ of tree contexts of type $\alpha$ for $\mathbb{T}(\Sigma)$ as the smallest set of terms such that $\lambda x_{1} \ldots x_{n} . t$ is in $\mathbb{T}_{[]}^{\alpha}(\Sigma)$ if $\mathbf{X}=\left\{x_{1}, \ldots, x_{n}\right\}$ is a typed alphabet, where for every $1 \leq i \leq n, \tau\left(x_{i}\right)=\alpha_{i}, \alpha=\alpha_{1} \rightarrow \ldots, \rightarrow \alpha_{n} \rightarrow o$, and $t \in \mathbb{T}(\Sigma \cup \mathbf{X})$.

The set of tree contexts on $(\Sigma)$ is defined by $\left(\mathbb{T}_{[]}^{\alpha}(\Sigma)\right)_{\alpha \in \mathscr{T}_{o}}$. Finally, such a tree context $\lambda x_{1} \ldots x_{n} . t$ is said:

- linear if $|t|_{x_{i}}=1$ for every $1 \leq i \leq n$

- almost affine iffor every $1 \leq i \leq n,|t|_{x_{i}}>1$ iff $x_{i} \in \Sigma^{1} \cup \mathbf{X}^{1}$

Remark 1. In what follows, we will note trees by $t, t^{\prime}, t_{1}, \ldots$, contexts by $C, C^{\prime}, C_{1}, \ldots$. Moreover, finite sequences of variables $x_{1}, \ldots, x_{n}$ will often be written as $\bar{x}$, and identically, finite sequences of contexts of the form $C_{1}, \ldots, C_{m}$ will be noted by $\bar{C}$. 
It is important to remark that $\mathbb{T}(\Sigma)=\mathbb{T}_{[]}^{o}(\Sigma)$. Moreover, tree contexts are defined as particular simply-typed $\lambda$-terms. In the rest of the document, we will take as granted the usual notions of substitutions, $\alpha$-conversion, $\beta$-reduction and $\eta$-conversions defined in the simply-typed $\lambda$-calculus (see [Bar84,SU06] for details). These notions will be used to define a tree context substition: given a typed alphabet $\mathbf{X}, \sigma=\left[x_{1}:=C_{1}, \ldots, x_{n}:=C_{n}\right]$ (where $x_{i} \in \mathbf{X}$ and $C_{i} \in \mathbb{T}_{[]}^{\tau\left(x_{i}\right)}(\Sigma)$ for every $i \in\{1, \ldots, n\}$ ) is a term substitution from $\mathbf{X}$ to $\mathbb{T}_{[]}(\Sigma)$; the application of $\sigma$ to a tree context $C$ is defined as $|C \cdot \sigma|_{\beta}$, i.e. the normal form of the application of the $\lambda$-term substitution $\sigma$ to the $\lambda$-term $C$. Finally, we define the application of tree contexts $C_{1}, \ldots, C_{n}$ to a tree context $\lambda x_{1} \ldots x_{n}$.t by $\operatorname{app}\left(C, C_{1}, \ldots, C_{n}\right)=\left|C \cdot\left[x_{1}:=C_{1}, \ldots, x_{n}:=C_{n}\right]\right|_{\beta}$.

Definition 8. An alphabet $\Sigma$ is said multi-typed if to every element a in $\Sigma$ is associated a natural number $n \in \mathbb{N}$, called the rank of a (written $r(a)$ ), and to every pair $(a, i)$, where $a \in \Sigma$ and $1 \leq i \leq r(a)$ is associated a type in $\mathscr{T}_{o}$ (written $\tau(a, i)$ )

We are in position of defining MCFTGs. These grammars can be seen as MCFGs on tree contexts.

Definition 9. A multiple context-free tree grammar (MCFTG for short) is a tuple $G=$ $(N, \Sigma, \mathbf{Y}, P, S)$ where:

- $N$ is a finite multi-typed alphabet of elements called non-terminals.

- $\Sigma$ is a finite second-order alphabet of elements called terminals.

- Y is an enumerable typed alphabet of variables disjoint from $\Sigma$.

- $S \in N$ is called the starting non-terminal of $G$ and verifies $r(S)=1$.

- $P$ is a finite set of production rules of the form:

$$
A_{0}\left(C_{1}, \ldots, C_{r_{0}}\right) \rightarrow A_{1}\left(x_{1,1}, \ldots, x_{1, r_{1}}\right), \ldots, A_{n}\left(x_{n, 1}, \ldots, x_{n, r_{n}}\right)
$$

where

- for very $0 \leq i \leq n, r\left(A_{i}\right)=r_{i}$.

- for every $1 \leq k \leq r, C_{k} \in \mathbb{T}_{[]}^{\alpha_{i}}(\Sigma \cup \mathbf{Y})$, where $\alpha_{i}=t\left(A_{0}, k\right)$.

- for every $i \in\{1, \ldots, n\}$ and $j \in\left\{1, \ldots, r_{i}\right\}, x_{i, j} \in \mathbf{Y}$ is of type $t\left(A_{i}, j\right)$ and verifies $\Sigma_{1 \leq k \leq r_{0}}\left|C_{k}\right|_{x_{i}, j}=1$. Moreover, given $i_{1}, i_{2} \in\{1, \ldots, n\}$ and $j_{1} \in\left\{1, \ldots, r_{i_{1}}\right\}, j_{2} \in$ $\left\{1, \ldots, r_{i_{2}}\right\}$, we have $x_{i_{1}, j_{1}}=x_{i_{2}, j_{2}}$ iff $i_{1}=i_{2}$ and $j_{1}=j_{2}$

Such a grammar is said linear (resp. almost affine) if for every rule $\pi \in P$ of the form $A_{0}\left(C_{1}, \ldots, C_{r_{0}}\right) \rightarrow A_{1}\left(x_{1,1}, \ldots, x_{1, r_{1}}\right), \ldots, A_{n}\left(x_{n, 1}, \ldots, x_{n, r_{n}}\right)$, the contexts $C_{1}, \ldots, C_{r_{0}}$ are linear (resp. almost affine).

Example 1. Let us consider the MCFTG $G=(N, \Sigma, \mathbf{Y}, P, S)$ where $N=\left\{S, A_{1}, A_{2}\right\}, \Sigma=$ $\{a, b, c, d, \epsilon, e, f\}$, and $P$ is made of the following production rules:

$$
\begin{aligned}
& S\left(e x_{1} x_{2} x_{3} x_{4}\right) \rightarrow A_{1}\left(x_{1}, x_{3}\right), A_{2}\left(x_{2}, x_{4}\right) \\
& A_{1}(\epsilon, \epsilon) \\
& A_{1}\left(f a x_{1}, f c x_{3}\right) \rightarrow A_{1}\left(x_{1}, x_{3}\right) \\
& A_{1}(\epsilon, \epsilon) \\
& A_{1}\left(f b x_{2}, f d x_{4}\right) \rightarrow A_{1}\left(x_{2}, x_{4}\right)
\end{aligned}
$$

This MCFTG is a linear MCFTG. Moreover, $r(S)=1, r\left(A_{1}\right)=r\left(A_{2}\right)=2$ and $\tau(S, 1)=$ $\tau\left(A_{1}, 1\right)=\tau\left(A_{1}, 2\right)=\tau\left(A_{2}, 1\right)=\tau\left(A_{2}, 2\right)=o$. 
Definition 10. Given a $M C F T G G=(N, \Sigma, \mathbf{Y}, P, S)$, and a non-terminal $A \in N$ of arity $r$, we define the relation of derivation $\rightarrow_{G}^{*}$ by:

1. if $A\left(C_{1}, \ldots, C_{r}\right)$ is a production rule in $P$, then $\rightarrow_{G}^{*} A\left(C_{1}, \ldots, C_{n}\right)$

2. $\rightarrow{ }_{G}^{*} A\left(C_{1} \cdot \sigma, \ldots, C_{r} \cdot \sigma\right)$ if:

- $\sigma$ is a substitution from $\mathbf{Y}$ to $\mathbb{T}_{[]}(\Sigma)$

- there exists a rule $A\left(C_{1}, \ldots, C_{r}\right) \rightarrow A_{1}\left(x_{1,1}, \ldots, x_{1, r_{1}}\right), \ldots, A_{n}\left(x_{n, 1}, \ldots, x_{n, r_{n}}\right)$ in $P$

- for every $1 \leq i \leq n$, the relation $\rightarrow_{G}^{*} A_{i}\left(x_{1,1} \cdot \sigma, \ldots, x_{i, r_{i}} \cdot \sigma\right)$ is verified.

The language $L(G)$ generated by a MCFTG $G=(N, \Sigma, \mathbf{Y}, P, S)$ is defined as the set $\left\{t \in \mathbb{T}_{[]}(\Sigma) \mid \rightarrow_{G}^{*} S(t)\right\}$. The class of tree languages MCFTL is defines as the smallest set which contains every language derived by some MCFTG.

In the previous example, the language $L(G)$ derived by the MCFTG $G$ is

$$
\{c(\underbrace{f a(f a \ldots f a}_{m} \epsilon) \ldots)(\underbrace{f b(f b \ldots f b}_{n} \epsilon) \ldots)(\underbrace{f c(f c \ldots f c}_{m} \epsilon) \ldots)(\underbrace{f d(f d \ldots f d}_{m} \epsilon) \ldots) \mid n, m \in \mathbb{N}\}
$$

Moreover, we can remark that $y L(G)=\left\{a^{m} b^{n} c^{m} d^{n} l n, m \in \mathbb{N}\right\}$ which is known to be a MCFL. This result is generalized in the following theorem:

Theorem 1 ([dGP04,Kan06]). A language L belongs to MCFL iff there is a linear $M C T F G G$ such that $y L(G)=L$.

Finally, we give the following theorem related to the complexity of the recognition problem of tree languages derived by a MCFTG.

Theorem 2 ([Kan07,Yos06], [BS11]). The recognition problem of an almost affine MCFTG is LOGCFL.

Because the class of problems LOGCFL is a sub-class of the problem solvable in polynomial-time, this theorem will be of particular importance so as to prove that the new class of language we define in this article is recgnizable in polynomial time, and is hence included in MCSL.

\section{IO-substitutions, semilinearity and constant-growth}

\subsection{IO-substitution and copies}

In this section, we introduce a new operation on languages, which we will use to define constant-growth languages. This operation, which we call IO-substitution enables a specific kind of copying operation. Thanks to this operation, we want to capture some languages which do not belong to MCFL, but still verify the constant-growth property, as shown in [Kal10]:

Example 2. The language $L_{a, b}\left\{\left(a^{n} b^{n}\right)^{m} \mid n, m \in \mathbb{N}-\{0\}\right\}$ is not a MCFL. Nevertheless, it is a semilinear language, as its Parikh's image is $\{(1,1)+k(1,1) \mid k \in \mathbb{N}\}$.

The language $L_{\text {count }}=\left\{\left(a^{n} d\right)^{m} a^{n} \mid n, m \in \mathbb{N}\right\}$ is not a semilinear language, but verifies the constant-growth property.

The language $L_{\text {exp }}=\left\{a^{2^{n}} \mid n \in \mathbb{N}\right\}$ is neither a semilinear, nor a language which verifies the constant-growth property. 
Interestingly, those languages are also known to be generated by IO-macro grammars [Fis68b,Fis68a], in which copying operations are allowed in a specific way, which we will not discuss in detail in this article. In brief words, Fischer makes a clean distinction between IO derivations, in which every copies of the same occurrence of a non-terminal are replaced using the same rewriting rule, and an OI derivation, in which such a constraint does not exist. For instance, let us consider a word $w=\alpha_{1} A \alpha_{2} A \alpha_{3}$, where $A$ does not appear in $\alpha_{1} \alpha_{2} \alpha_{3}$, and the two occurrences of $A$ are supposed to result from a copying operation. We consider the rewritings of $A$ as $w_{1}$ or $w_{2}$. An IO derivation forces the two occurrences of $A$ to be rewritten using the same rewriting rule; it hence defines the language $\left\{\alpha_{1} w \alpha_{2} w \alpha_{3} \mid w \in\left\{w_{1}, w_{2}\right\}\right\}$. An OI derivation would lead to the derivation of the language $\left\{\alpha_{1} w_{1}^{\prime} \alpha_{2} w_{2}^{\prime} \alpha_{3} \mid w_{1}^{\prime}, w_{2}^{\prime} \in\left\{w_{1}, w_{2}\right\}\right\}$. We use the same ideas to define the IO-substitution on languages, as contrasted with the classic susbstitution, which can be seen as an OI-substitution.

Definition 11. Let us consider an alphabet $\Sigma$ and a variable $x$, and two languages $L_{1} \in(\Sigma \cup\{x\})^{*}$ and $L_{2} \in \Sigma^{*}$. Given words $w_{1} \in L_{1}, w_{2} \in L_{2}$ and $x \in \chi$, we define the word $w_{1}\left[x:=w_{2}\right]_{I O} \in \Sigma$ by induction:

$-x\left[x:=w_{2}\right]_{I O}=w_{2}$

$-a\left[x:=w_{2}\right]_{I O}=a$ if $a \neq x$

$-w w^{\prime}\left[x:=w_{2}\right]_{I O}=w\left[x:=w_{2}\right]_{I O} w^{\prime}\left[x:=w_{2}\right]_{I O}$

The language $L_{1}\left[x:=L_{2}\right]_{I O}$ is then defined as $\left\{w_{1}\left[x:=w_{2}\right]_{I O} \in \Sigma \mid w_{1} \in L_{1}, w_{2} \in L_{2}\right\}$.

Based on this operation, we can build the languages $L_{a, b}$ and $L_{c o u n t}$ easily from context-free languages. Indeed, given $L_{1}=\left\{x^{n} \mid n \in \mathbb{N}-\{0\}\right\}$ and $L_{2}=\left\{a^{m} b^{m} \mid m \in\right.$ $\mathbb{N}-\{0\}\}$, the language $L_{a, b}$ is the language $L_{1}\left[x:=L_{2}\right]_{I O}$. In the same way, $L_{\text {count }}=$ $L_{1}^{\prime}\left[x:=L_{2}^{\prime}\right]_{I O}$, where $L_{1}^{\prime}=\left\{(x d)^{m} x \mid m \in \mathbb{N}\right\}$ and $L_{2}^{\prime}=\left\{a^{n} \mid n \in \mathbb{N}\right\}$. On the other hand, there is no trivial way to use the IO-substitution operation on context-free languages or mildly context-sensitive languages to generate $L_{\text {exp }}$. One can also remark that $L_{1}, L_{2}, L_{1}^{\prime}$ and $L_{2}^{\prime}$ are context-free languages, and are therefore semilinear languages (hence, verifiy the constant-growth property). It is therefore natural to investigate the conditions under which semilinearity and the constant-growth propery are preserved by the IOsubstitution operation.

\subsection{Preserving the semilinearity and constant-growth properties}

In the previous examples, we have seen that the newly introduced operation of IOsubstitution allows, in some cases, building semilinear languages or constant-growth languages from semilinear languages. We now investigate the conditions which allow preserving these properties.

Definition 12. Let us consider a semilinear language $L \subseteq \Sigma^{*}$ and its Parikh image $p(L)=\bigcup_{i \in I} S_{i}(I \subset \mathbb{N}$ finite $)$, where for every $i \in I$,

$$
S_{i}=\left\{\overrightarrow{v_{i, 0}}+n_{1} \overrightarrow{v_{i, 1}}+\ldots+n_{r_{i}} \overrightarrow{v_{i, r_{i}}} \mid n_{1}, \ldots, n_{r_{i}} \in \mathbb{N}\right\}
$$

Given $a \in \Sigma, L$ is said a-isolating if, for every $i \in I$, there exists a unique $l_{i} \in\left\{0, \ldots, r_{i}\right\}$ such that: 
- for every $j \in\left\{0, \ldots r_{l}\right\}, \overrightarrow{v_{i, j}}\left(k_{a}\right) \neq 0$ iff $j=l_{i}$.

- for every $c \in \Sigma-\{a\}, \overrightarrow{v_{i, l_{i}}}\left(k_{c}\right)=0$

Example 3. The language $\left\{x^{n} \mid n \in \mathbb{N}\right\}$ is obviously $x$-isolating. The language $a b x(a)^{*} x^{*}$ is not $x$-isolating as its Parikh image is $\{(1,1,1)+n(1,0,0)+m(0,0,1) \mid n, m \in \mathbb{N}\}$. Finally, the language $(x a)^{*} x$ is not $x$-isolating, its Parikh image being $\{(0,1)+n(1,1) \mid n \in \mathbb{N}\}$.

Remark 2. Given a language $L \in \Sigma^{*}$ and $a \in \Sigma$ such that $L$ is $a$-isolating, each linear set $S \in\left\{S_{1}, \ldots, S_{l}\right\}$, such that $\bigcup_{i=1}^{l} S_{i}=p(L)$ can be written as:

$$
\sum_{i \in I} n_{i} \overrightarrow{x_{i}}+n_{t} \vec{x}_{t}
$$

where $n_{0}=1, t \notin I$, for every $i \in I \cup\{t\} \vec{x}_{i}\left(k_{a}\right) \neq 0$ iff $i=t$, and $\vec{x}_{t}\left(k_{c}\right)=0$ for every $c \in \Sigma-\{a\}$.

We now show that the IO-substitution of a letter $a$ by a semilinear language, on a $a$-isolating language generates a semilinear language.

Theorem 3. Given a semilinear language $L_{1} \in(\Sigma \cup\{x\})^{*}, x$-isolating, and a semilinear language $L_{2}$, the language $L_{1}\left[x:=L_{2}\right]_{\text {IO }}$ is semilinear.

Proof. Let us consider the language $L=L_{1}\left[x:=L_{2}\right]_{I O}$, and a word $w \in L$. By definition, there exist $w_{1} \in L_{1}$ and $w_{2} \in L_{2}$ such that $w=w_{1}\left[x:=w_{2}\right]_{I O}$. Because $L_{1}$ and $L_{2}$ are semilinear, for $p\left(L_{1}\right)$ and $p\left(L_{2}\right)$ the Parikh images of $L_{1}$ and $L_{2}$ respectively, there exist linear sets $S_{1} \subset p\left(L_{1}\right)$ and $S_{2} \subset p\left(L_{2}\right)$ such that $p\left(w_{1}\right) \in S_{1}$ and $p\left(w_{2}\right) \in S_{2}$; hence:

- $p\left(w_{1}\right) \in\left\{\overrightarrow{x_{0}}+n_{1} \overrightarrow{x_{1}}+\ldots+n_{p} \overrightarrow{x_{p}} \mid n_{1}, \ldots, n_{p} \in \mathbb{N}\right\}$

$-p\left(w_{2}\right) \in\left\{\overrightarrow{y_{0}}+m_{1} \overrightarrow{y_{1}}+\ldots+m_{r} \overrightarrow{y_{r}} \mid m_{1}, \ldots, m_{r} \in \mathbb{N}\right\}$

Moreover, because $L_{1}$ is $x$-isolating, we can write

$$
p\left(w_{1}\right) \in\left\{\sum_{i \in I} n_{i} \vec{x}_{i}+n_{t} \overrightarrow{x_{t}} \mid \text { for all } i \in I \cup\{t\}, n_{i} \in \mathbb{N} \text { and } n_{0}=1\right\}
$$

where $t \notin I, I \cup\{t\}=\{0, \ldots, p\}, \vec{x}_{i}\left(k_{x}\right)=0$ for every $i \in I$, and $\vec{x}_{t}\left(k_{c}\right)=0$ for every $c \in \Sigma$.

Then, by construction, the Parikh image of $w$ can be written as

$$
p(w)=\overrightarrow{z_{0}}+n_{1} \overrightarrow{z_{1}}+\ldots+n_{p} \overrightarrow{z_{p}}
$$

where, for every $i \in\{0, \ldots, p\}, \overrightarrow{z_{i}}=\vec{x}_{i}+\vec{x}_{i}\left(k_{x}\right) \vec{p}\left(w_{2}\right)$. Let us write $\vec{x}_{i}\left(k_{x}\right)=K_{i}$, for every $i \in\{0, \ldots, p\}$

The Parikh image of $w$ can be rewritten according to the following results:

1. $\overrightarrow{z_{i}}=\vec{x}_{i}$, for every $i \in I$, because $K_{i}=0$

2. $\overrightarrow{z_{t}}=K_{t} \vec{p}\left(w_{2}\right)$, because $\vec{x}_{t}\left(k_{a}\right)=0$ for every $a \neq x$. Hence,

$$
\begin{aligned}
\overrightarrow{z_{t}} & =K_{t}\left(\overrightarrow{y_{0}}+m_{1} \overrightarrow{y_{1}}+\ldots+m_{r} \overrightarrow{y_{r}}\right) \\
& =K_{t} \overrightarrow{y_{0}}+m_{1} K_{t} \overrightarrow{y_{1}}+\ldots+m_{r} K_{t} \overrightarrow{y_{r}}
\end{aligned}
$$


Finally,

$$
\begin{aligned}
p(w) & \in\left\{\Sigma_{i \in I} n_{i} \overrightarrow{x_{i}}+n_{t} \Sigma_{j \in\{1, \ldots, r\}} m_{j} K_{t} \overrightarrow{y_{j}} \mid \forall i \in\{1, \ldots, r\}, j \in I \cup\{t\}, m_{i}, n_{j} \in \mathbb{N}\right\} \\
& \in\left\{\Sigma_{i \in I} n_{i} \vec{x}_{i}+n_{t} \Sigma_{j \in\{1, \ldots, r\}} m_{j} \overrightarrow{y_{j t}} \mid \forall i \in\{1, \ldots, r\}, j \in I \cup\{t\}, m_{i}, n_{j} \in \mathbb{N}\right\} \\
& \in\left\{\Sigma_{i \in I} n_{i} \vec{x}_{i}+\Sigma_{j \in\{1, r\}} m_{j} \vec{y}_{j t}^{\prime} \mid \forall i \in\{1, \ldots, r\}, j \in I \cup\{t\}, m_{i}, n_{j} \in \mathbb{N}\right\}
\end{aligned}
$$

belongs to a linear set.

Moreover, for $w^{\prime}=w_{1}^{\prime}\left[x:=w_{2}^{\prime}\right]_{I O}$, where $\vec{p}\left(w_{1}^{\prime}\right)$ and $\vec{p}\left(w_{2}^{\prime}\right)$ belong to the same linear sets as $\vec{p}\left(w_{1}\right)$ and $\vec{p}\left(w_{2}\right)$, it is easy to verify that $\vec{p}\left(w^{\prime}\right)$ belongs to the same linear set as $\vec{p}(w)$. The set of linear sets built in the proof is trivially finite because $\vec{p}\left(L_{1}\right)$ and $\vec{p}\left(L_{2}\right)$ are finite. Hence $L$ is a semilinear language.

We now show that the preservation of the constant-growth property is more direct, as substituting a letter by a constant-growth language, in a constant-growth language suffices.

Theorem 4. Given a constant-growth language $L_{1} \subseteq(\Sigma \cup\{x\})^{*}$, and a constant-growth language $L_{2} \subseteq \Sigma^{*}$. The language $L_{1}\left[x:=L_{2}\right]_{\text {Io }}$ is constant-growth.

Proof. Let us a consider a word $w_{1}$ in $L_{1}$, and a word $w_{2}$ in $L_{2}$. We note by $k$ the number of occurrences of $x$ in $w_{1}$ (i.e. $\vec{p}\left(w_{1}\right)\left(k_{x}\right)=k$ ).

By definition of a constant-growth language, for every $i \in\{1,2\}$, there exists a constant $c_{i} \in \mathbb{N}$ such that, for every word $w_{i}^{\prime} \in L_{i}$ verifying $\left|w_{i}^{\prime}\right| \geq c_{i}$, there exist $\vec{x}, \vec{y} \in \mathbb{N}^{|\Sigma|}$ such that $\vec{p}\left(w_{i}^{\prime}\right)=\vec{x}+\vec{y}$ and, for every $k \geq 1, \vec{x}+k \vec{y} \in \vec{p}\left(L_{i}\right)$.

Let us first suppose $\left|w_{2}\right| \geq c_{2}$. Then $\vec{p}\left(w_{1}\left[x:=w_{2}\right]_{I O}\right)=\vec{z}+k \overrightarrow{p^{\prime}}\left(w_{2}\right)=\vec{z}+k \overrightarrow{x_{2}^{\prime}}+k \overrightarrow{y_{2}^{\prime}}$, where $\vec{z}$ is the Parikh image of $w_{1}$ on $\Sigma$, and $\overrightarrow{p^{\prime}}\left(w_{2}\right)$ is the Parikh image of $w_{2}$ on the same alphabet. By hypothesis, we can consider an integer $i \geq 1$ and a word $w_{2}^{\prime} \in L_{2}$ such that $\vec{p}\left(w_{2}^{\prime}\right)=\overrightarrow{x_{2}}+i \overrightarrow{y_{2}}$. Then, $\vec{p}\left(w_{1}\left[x:=w_{2}^{\prime}\right]_{I O}\right)=\vec{z}+k \overrightarrow{p^{\prime}}\left(w_{2}^{\prime}\right)=\vec{z}+k \overrightarrow{x^{\prime}}+k \overrightarrow{i y^{\prime}}$.

Otherwise, suppose $\left|w_{2}\right|<c_{2}$ and $\left|w_{1}\right| \geq c_{1}$. Then $\vec{p}\left(w_{1}\right)=x_{1}+y_{1}$, and given $i>1$, there exist a word $w_{1}^{\prime} \in L_{1}$ such that $\vec{p}\left(w_{1}^{\prime}\right)=\overrightarrow{x_{1}}+i \overrightarrow{y_{1}}$. We give $k_{1}=\overrightarrow{x_{1}}(x)$ and $k_{2}=\overrightarrow{y_{1}}(x)$. Then

$$
\begin{aligned}
& -\vec{p}\left(w_{1}\left[x:=w_{2}\right]_{I O}\right)=\overrightarrow{x_{1}^{\prime}}+k_{1} \overrightarrow{p^{\prime}}\left(w_{2}\right)+\overrightarrow{y_{1}^{\prime}}+k_{2} \overrightarrow{p^{\prime}}\left(w_{2}\right) \\
& -\vec{p}\left(w_{1}^{\prime}\left[x:=w_{2}\right]_{I O}\right)=\overrightarrow{x_{1}^{\prime}}+k_{1} \overrightarrow{p^{\prime}}\left(w_{2}\right)+i y_{1}^{\prime}+i k_{2} \overrightarrow{p^{\prime}}\left(w_{2}\right)
\end{aligned}
$$

where given $\vec{x}$ a Parikh vector on $\Sigma \cup\{x\}, \overrightarrow{x^{\prime}}$ is the same vector on $\Sigma$; and $\overrightarrow{p^{\prime}}\left(w_{2}\right)$ is the Parikh image of $w_{2}$ on the same alphabet.

As a conclusion, $L_{1}\left[w:=L_{2}\right]_{I O}$ is a constant-growth language. We can consider the constant associated to the growth of this language as $c_{1} c_{2}$.

Thanks to the two previous theorems, we proved that the constant-growth and semilinearity properties of languages can be preserved by the IO-substitution operation. In the following section, we seek a class of languages bigger than MCFL which is included in MCSL, by using this operation. 


\section{IO-Multiple Context-Free Languages}

\subsection{Definition}

In the previous section, we proved some properties on the IO-substitution which allows preserving the semilinearity or constant-growth properties. As mentionned in the first section, we can consider the class of multiple context-free languages as the biggest class approximating mildly context-sensitive languages. We now extend the class MCFL by using the IO-substition operation.

Definition 13. We consider the family of IO-mildly context sensitive languages (written $\mathbf{I O}-\mathbf{M C F L}$ ) as the smallest family such that $L \in \mathbf{I O}-\mathbf{M C F L}$ if:

- $L \in$ MCFL or

- if $L=L_{1}\left[x:=L_{2}\right]_{\text {IO }}$ where $L_{1}, L_{2} \in \mathbf{I O}$-MCFL.

Thanks to this definition, languages $L_{a, b}$ and $L_{\text {count }}$ can be considered to belong to IO-MCFL. The following theorem is a direct corrolary of Theorem 4.

Theorem 5. Every language L in IO-MCFL verifies the constant-growth property.

Proof. We proceed by induction on $L$. If $L \in M C F L$, the result is given by the fact that $L$ is semilinear. Otherwise, there exist $L_{1} \in$ IO-MCFL and $L_{2} \in$ IO-MCFL such that $L=L_{1}\left[x:=L_{2}\right]_{I O}$. By induction hypothesis, $L_{1}$ and $L_{2}$ verify the constant-growth property. According to Theorem $4, L$ is constant-growth.

In order to see if IO-MCFL is a better approximation of the class MCSL, we then need to know if such languages are tractable in polynomial time. In order to do so, we give a formalism which exactly captures the IO-MCFL class of languages, thanks to the multiple context-free tree languages given in section 2.2.

\subsection{IO-MCFGs as quasi-affine MCFTGs}

As mentionned in section 2.2, the class MCFTL is connected to MCFL through theorem 1. In what follows, we seek the characterization of some MCFTGs which yield are exactly IO-MCFLs.

Intuitively, given a language $L_{1} \in \Sigma \cup\{x\}$, where $L_{1}$ and $L_{2}$ are MCFLs, we can remark that the letter $x$ may have many occurrences in $w_{1} \in L_{1}$. Given a MCFTG $G_{1}$ such that $y L\left(G_{1}\right)$, the idea is too consider $x$ not as a leaf in the trees derived by $G_{1}$, but by a variable which can be substituted by a word $w_{2} \in L_{2}$. Therefore, we need to build a MCFTG $G_{1}^{\prime}$ such that $t \in L\left(G_{1}\right)$ iff $\lambda x . t \in L\left(G_{1}^{\prime}\right)$, and then apply a simple $S\left(x_{1} x_{2}\right) \rightarrow S_{1}^{\prime}\left(x_{1}\right), S\left(x_{2}\right)$ where $S_{1}^{\prime}$ and $S_{2}$ are the starting non-terminals of $G_{1}^{\prime}$ and $G_{2}$ respectively.

Remark that, because $x$ will now be considered as a variable, the contexts appearing in the left-hand side of a production rule in $G_{1}^{\prime}$ will not be linear anymore. But $x$ being a leaf, its type is $\tau(x)=o$, and the contexts will therefore be almost affine, which still ensures the recognizability problem belongs to LOGCFL, hence to the class of problems solvable in polynomial-time. 
Definition 14. Given a MCFTG $G=(N, \Sigma \cup\{x\}, \mathbf{Y}, P, S)$ where $\tau(x)=\alpha$, we define the $M C F T G$

$$
\operatorname{abs}(G, x)=(\operatorname{abs}(N, x), \Sigma, a b s(\mathbf{Y}, x), a b s(P, x), a b s(S, x))
$$

as follows:

- given a type $\alpha=\alpha_{1} \rightarrow \ldots \rightarrow \alpha_{n} \rightarrow \alpha_{0}$ in $\mathscr{T}_{o}$, we define abs $(\alpha, x)=\tau(x) \rightarrow \alpha_{1} \rightarrow$ $\ldots \rightarrow \alpha_{n} \rightarrow \alpha_{0}$.

- for every $A \in N$, abs $(A, x)=A$ such that $r\left(A^{\prime}\right)=r(A)$ and for every $1 \leq i \leq r(A)$, $t\left(A^{\prime}, i\right)=\operatorname{abs}(t(A, i), x)$. Finally, abs $(N, x)=\{a b s(A, x) \mid A \in N\}$.

$-y \in \mathbf{Y}$ and $\tau(y)=\gamma$ in $\mathbf{Y}$ iff $y \in \operatorname{abs}(\mathbf{Y}, x)$ and $\tau(y)=\operatorname{abs}(y, x)$ in $\operatorname{abs}(\mathbf{Y}, x)$.

- given a tree context $C$ in $\mathbb{T}_{[]}(\Sigma \cup \mathbf{Y})$, we define abs $(C, x) \in \mathbb{T}_{[]}(\Sigma \cup$ abs $(\mathbf{Y}, x))$, as $\lambda x . \operatorname{abs}(C, x)$, where:

- $\operatorname{abs}\left(\lambda y \cdot C^{\prime}, x\right)=\lambda y \cdot \operatorname{abs}\left(C^{\prime}, x\right)$, for $\left.C^{\prime} \in \mathbb{T}_{[]}(\Sigma \cup \mathbf{Y}\}\right)$

- $\operatorname{abs}(c, x)=c$ for $c \notin \mathbf{Y}$

- $\operatorname{abs}(y, x)=(y x)$, for $y \in \mathbf{Y}$.

- $\operatorname{abs}\left(t_{1} t_{2}, x\right)=a b s\left(t_{1}, x\right) a b s\left(t_{2}, x\right)$.

Finally a rule

$$
A_{0}\left(C_{1}, \ldots, C_{r}\right) \rightarrow A_{1}\left(x_{1,1}, \ldots, x_{1, r_{1}}\right), \ldots, A_{n}\left(x_{n, 1}, \ldots, x_{n, r_{n}}\right)
$$

belongs to P iff

$$
A_{0}^{\prime}\left(C_{1}^{\prime}, \ldots, C_{r}^{\prime}\right) \rightarrow A_{1}^{\prime}\left(x_{1,1}^{\prime}, \ldots, x_{1, r_{1}}^{\prime}\right), \ldots, A_{n}^{\prime}\left(x_{n, 1}^{\prime}, \ldots, x_{n, r_{n}}^{\prime}\right)
$$

belongs to abs $(P, x)$, where $A_{i}^{\prime}=\operatorname{abs}\left(A_{i}, x\right)$ and $x_{i, j}^{\prime}=\operatorname{abs}\left(x_{i, j}, x\right)$ for every $0 \leq i \mid$ eqn and $1 \leq j \leq r_{i}$, and $C_{k}^{\prime}=\operatorname{abs}\left(C_{k}, x\right)$ for every $1 \leq k \leq r$.

The transformation given by abs intuitively result in considering $x$ as a variable, on which tree context substitutions can be applied. This is made explicit in the following theorem.

Lemma 1. Given a MCFTG $G=(N, \Sigma \cup\{x\}, \mathbf{Y}, P, S)$, the language $L(\operatorname{abs}(G, x))$ is equal to $\left\{\lambda x . C \in \mathbb{T}_{[]}(\Sigma) \mid C \in L(G)\right\}$.

Proof. We consider the general form of a MCFTG so that the language derived is a tuple of tree contexts, and We proceed by induction on the derivation of such a tuple $\bar{C} \in L$. Let us consider the last rule $\pi$ used to derive $\bar{C}$ in $G$, which general form is:

$$
A_{0}\left(C_{1}, \ldots, C_{r}\right) \rightarrow A_{1}\left(\overline{x_{1}}\right), \ldots, A_{n}\left(\overline{x_{n}}\right)
$$

If $n=0$, then $\bar{C}=\left(C_{1}, \ldots, C_{r}\right)$ and $\operatorname{abs}(\pi, x)=\operatorname{abs}\left(A_{0}, x\right)\left(C_{1}^{\prime}, \ldots, C_{r}^{\prime}\right)$, where for every $1 \leq i \leq r, C_{i}^{\prime}=\operatorname{abs}\left(C_{i}, x\right)$. Moreover, $C_{i}^{\prime} \in \mathbb{T}_{[]}(\Sigma)$ and $C_{i}^{\prime}=\lambda x . C_{i}$ by construction. The tuple $\left(\lambda x . C_{1}, \ldots, \lambda x . C_{r}\right)$ is therefore recognized by abs $(G, x)$.

If $n \neq 0$; by induction hypothesis, for every $1 \leq k \leq n$, the following statement stands: $\rightarrow_{G}^{*} A_{k}\left(C_{k, 1}, \ldots, C_{k, r_{k}}\right)$ iff $\rightarrow_{\text {abs }(G, x)}^{*}$ abs $\left(A_{k}, x\right)\left(\lambda x . C_{k, 1}, \ldots, \lambda x . C_{k, r_{k}}\right)$. Therefore, given a tree context substitution $\sigma=\left[x_{1,1}:=C_{1,1}, \ldots, x_{1, r_{1}}:=C_{1, r_{1}}, \ldots, C_{n, 1}, \ldots, x_{n, r_{n}}:=C_{n, r_{n}}\right]$, such that $\bar{C}=\left(C_{1} \cdot \sigma, \ldots, C_{r} \cdot \sigma\right)$, we build $\sigma^{\prime}=\left[x_{1,1}^{\prime}:=\lambda x \cdot C_{1,1}^{\prime}, \ldots, x_{n, r_{n}}^{\prime}:=\lambda x . C_{n, r_{n}}^{\prime}\right]$ where $x_{i, j}^{\prime}:=\operatorname{abs}\left(x_{i, j}, x\right)$ and $C_{i, j}^{\prime}:=\operatorname{abs}\left(C_{i, j}, x\right)$, for every $1 \leq i \leq n$ and every $1 \leq j \leq r_{i}$. By construction, we obtain $\bar{C}=\left(\lambda x . C_{1}, \ldots, \lambda x . C_{r}\right)$. 
We now need to build the MCFTG which produces the substitution of $x$ in a tree context $C_{1}$ derived in abs $\left(G_{1}, x\right)$ by a tree context $C_{2}$ derived by $G_{2}$. In particular, if $\phi \operatorname{tau}(x)=o, x$ may appear in the yield of any tree derived by $G_{1}$, and replacing every occurrence of $x$ in $t_{1}=C_{1}$ by the tree $t_{2}=C_{2}$, resumes to $y t_{1}\left[x:=y t_{2}\right]_{I O}$.

First, we define disjunction of MCFTGs as follows: given two MCFTGs $G_{1}=$ $\left(N_{1}, \Sigma_{1}, \mathbf{Y}_{1}, P_{1}, S_{1}\right)$ and $G_{2}=\left(N_{2}, \Sigma_{2}, \mathbf{Y}_{\mathbf{2}}, P_{2}, S_{2}\right), G_{1}$ and $G_{2}$ are said disjoint if $N_{1} \cap$ $N_{2}=\emptyset$. Remark that such a relation implies $P_{1} \cap P_{2}=\emptyset$ and $S_{1} \neq S_{2}$.

Definition 15. A MCFTG $G$ is called an IO-MCFTG if

1. $G$ is a linear MCFTG or

2. there exist $G_{1}=\left(N_{1}, \Sigma \cup\{x\}, \mathbf{Y}_{1}, P_{1}, S_{1}\right)$ and $G_{2}=\left(N_{2}, \Sigma, \mathbf{Y}_{2}, P_{2}, S_{2}\right)$, two disjoint IO-MCFTGs, such that given $G_{1}^{\prime}=\left(N_{1}^{\prime}, \Sigma, \mathbf{Y}_{1}^{\prime}, P_{1}^{\prime}, S_{1}^{\prime}\right)=\operatorname{abs}\left(G_{1}, x\right), G=\left(N_{1}^{\prime} \cup N_{2} \cup\right.$ $\left.\{S\}, \Sigma, \mathbf{Y}_{1}^{\prime} \cup \mathbf{Y}_{2}, P_{1}^{\prime} \cup P_{2} \cup\{\pi\}, S\right)$ where:

(a) $S \notin N_{1}^{\prime} \cup N_{2}^{\prime}$ and $r(S)=1, t(S, 1)=o$.

(b) $\pi \notin P_{1}^{\prime} \cup P_{2}$ and $\pi=S\left(x_{1} x_{2}\right) \rightarrow S_{1}^{\prime}\left(x_{1}\right), S_{2}\left(x_{2}\right)$.

We now prove that this formalism exactly captures tree languages which yield form IO-MCFLs:

Theorem 6. A language $L$ is a IO-MCFL iff there exists a IO-MCFTG such that $L=y L(G)$.

Proof. We proceed by induction on $L$. If $L$ is a MCFL, there exists a linear MCFTG $G$ such that $y L(G)=L$, and $G$ si a IO-MCFTG by definition. Conversely, for $G$ a linear MCFTG, $y L(G)$ is a IO-MCFL. If there exist $L_{1} \subseteq(\Sigma \cup\{x\})^{*}$ and $L_{2} \subseteq \Sigma^{*}$ two MCFLs, such that $L=L_{1}\left[x:=L_{2}\right]_{I O}$, by induction hypothesis, there exist two MCFTGs $G_{1}=$ $\left(N_{1}, \Sigma \cup\{x\}, \mathbf{Y}_{1}, P_{1}, S_{1}\right)$ and $G_{2}=\left(N_{2}, \Sigma, \mathbf{Y}_{2}, P_{2}, S_{2}\right)$, which can be considered disjoint, and such that $L_{1}=y L\left(G_{1}\right)$ and $L_{2}=y L\left(G_{2}\right)$. According to Lemma $1, L\left(\operatorname{abs}\left(G_{1}, x\right)\right)=$ $\left\{\lambda x . t \in \mathbb{T}_{[]}^{o \rightarrow o}(\Sigma) \mid t \in L\left(G_{1}\right)\right\}$. We consider the grammar

$$
G=\left(\operatorname{abs}\left(N_{1}, x\right) \cup N_{2} \cup\{S\}, \Sigma, \operatorname{abs}\left(\mathbf{Y}_{1}, x\right) \cup \mathbf{Y}_{\mathbf{2}}, \operatorname{abs}\left(P_{1}, x\right) \cup P_{2}\{\pi\}, S\right)
$$

such that $S \notin \operatorname{abs}\left(N_{1}, x\right) \cup N_{2}$ and $\pi=S\left(x_{1} x_{2}\right) \cup \operatorname{abs}\left(S_{1}, x\right)\left(x_{1}\right), S_{2}\left(x_{2}\right)$. A $t$ tree is recognized by this grammar iff there exist $t_{1} \in L\left(G_{1}\right)$ and $t_{2} \in L\left(G_{2}\right)$ such that $t=\operatorname{app}\left(t_{1}, t_{2}\right)$ which is the result of substituting every occurrence of $x$ in $t_{1}$ by $t_{2}$. Finally, $y L(G)=$ $\left\{w_{1}\left[x:=w_{2}\right]_{I O} \in \Sigma \mid w_{1} \in L_{1}, w_{2} \in L_{2}\right\}$. Conversely, the yield of the language of an IOMCFTG is an IO-MCFL.

It now suffices to show that the recognizability problem in this formalism can be solved in polynomial-time, which is a direct consequence of the following lemma.

Lemma 2. If $G$ is an almost affine $M C F T G$, then $\operatorname{abs}(G, x)$ is an almost affine $M C F T G$.

Proof. In order to prove this property, it suffices to prove that, given an almost affine tree context $C$ in $\mathbb{T}_{[]}(\Sigma \cup\{x\}), \lambda x$.abs $(C, x)$ is a tree context in $\mathbb{T}_{[]}(\Sigma)$. Therefore, given the general form $\lambda x_{1}^{\alpha_{1}} \ldots x_{n}^{\alpha_{n}} . t$ of $C$, if a variable $\left.y^{\alpha} \notin \Sigma \cup\{x\}\right)$ verifies $|C|_{y} \neq 1$ then there exists $1 \leq j \leq n$ such that $y=x_{j}$ and $\alpha_{j}=o$. Moreover, $x$ might have a number of occurrences in $C$ different from 0 , which implies the same property is verified on $\lambda x . C$, which therefore remains almost affine. 
Corollary 1. The recognition problem of an IO-MCFL belongs to LOCGCFL.

As a conclusion, we proved that MCFL $\subsetneq$ IO-MCFL $\subseteq$ MCSL. This new class of languages can therefore be taken as the best approximation of the class of mildly context-sensitive languages.

While we proved that the recognition problem can be solved in polynomial-time, we next prove some usual properties taken to test the robustness of parsing in a given formalism, so as to explore additional proeprties of IO-MCFL.

\subsection{Closure properties}

In what follows, we study other properties enjoyed by IO-MCFLs. In particular, we are interested in the usual closure properties so as to know whether IO-MCFLs define a full abstract family of languages.

Definition 16. A family of languages $\boldsymbol{L}$ is called a (full)-abstract family of languages (written (full)-AFL) if $\boldsymbol{L}$ is closed by homomorphism, inverse homomorphism, intersection with a regular set, union, concatenation and the Kleene star.

In what follows, we show that most of this properties are verified.

Remark 3. A language $L \in M C F L$ can be written $L=L\left[x:=L^{\prime}\right]_{I O}$, where $x$ has no occurrence in $L$. The general form a IO-MCFL is therefore $L^{\prime}\left[x:=L^{\prime \prime}\right]_{I O}$

Definition 17. Given an alphabet $\Sigma$, a function $\mathscr{H}: \Sigma \mapsto \Sigma$ is called a renaming.

Lemma 3. The family IO-MCFL is closed by renaming.

Proof. Trivial.

The next closure properties are verified through direct proofs.

Theorem 7. The family IO-MCFL is closed by homomorphism, union, intersection, concatenation and the Kleene star.

Proof. Let us consider $L, L_{1}, L_{2} \in \mathbf{I O}-\mathbf{M C F L}$, languages built on the alphabet $\Sigma$ :

- we consider a homomorphism $\mathscr{H}: \Sigma^{*} \mapsto \Sigma^{*}$, and show that $\mathscr{H}(L) \in$ IO-MCFL by induction on $L$. If $L$ is a MCFL, the property is verified as the family of MCFLs is an AFL. Otherwise, there exist $L_{1}, L_{2} \in I O-M C L F$ such that $L=L_{1}\left[x:=L_{2}\right]_{I O}$; then $\mathscr{H}(L)=\mathscr{H}\left(\mathscr{L}[:=\mathscr{L}]_{\mathscr{I} O}\right)$. We built the $L_{1}^{\prime}=\mathscr{F}\left(L_{1}\right)$, where $\mathscr{F}$ is a symbol replacement such that $\mathscr{F}(x)=x^{\prime}$ and $\mathscr{H}\left(x^{\prime}\right)=x^{\prime} ; \mathscr{F}(a)=a$ otherwise. Then $L_{1}\left[x:=L_{2}\right]_{I O}=L_{1}^{\prime}\left[x^{\prime}:=L_{2}\right]_{I O}$, and $\mathscr{H}(\mathscr{L})=\mathscr{H}\left(L_{1}^{\prime}\left[x^{\prime}:=L_{2}\right]_{I O}\right)=\mathscr{H}\left(L_{1}^{\prime}\right)[x:=$ $\mathscr{H}\left(L_{2}\right)$ ]. By induction hypothesis, $\mathscr{H}\left(L_{1}^{\prime}\right), \mathscr{H}\left(L_{2}\right) \in$ IO-MCFL and by construction, $\mathscr{H}(L) \in \mathbf{I O}$-MCFL. 
- We show that $L_{1} \cup L_{2} \in \mathbf{I O}$-MCFL. We can consider the general form $L_{i}=L_{i}^{\prime}\left[x_{i}:=\right.$ $\left.L_{2}\right]_{I O}$, for $i \in\{1,2\}$. Moreover, $x_{1}$ and $x_{2}$ can be renamed so that $x_{1}$ (resp. $x_{2}$ ) has no occurrence in $L_{2}$ (resp. $L_{1}$ ). Then

$$
\begin{aligned}
L_{1} \cup L_{2} & =L_{1}^{\prime}\left[x_{1}:=L_{1}^{\prime \prime}\right]_{I O} \cup L_{2}^{\prime}\left[x_{2}:=L_{2}^{\prime \prime}\right]_{I O} \\
& =\left(\left(L_{1}^{\prime} \cup L_{2}^{\prime}\right)\left[x:=L_{1}^{\prime \prime}\right]_{I O}\right)\left[x:=L_{2}^{\prime \prime}\right]_{I O} \\
& =\left(\left(L_{1}^{\prime} \cup L_{2}^{\prime}\right)\left[x:=L_{2}^{\prime \prime}\right]_{I O}\right)\left[x:=L_{1}^{\prime \prime}\right]_{I O}
\end{aligned}
$$

and $L_{1}^{\prime} \cup L_{2}^{\prime}$ is a $I O-M C L F$ by induction hypothesis (the result is given in the case $L_{1}^{\prime}$ and $L_{2}^{\prime}$ are MCFLs), which implies $L_{1} \cup L_{2}$ is a IO-MCFL by construction.

- We show that $L_{1} \cap L_{2} \in \mathbf{I O}$-MCFL, in a similar way:

$$
\begin{aligned}
L_{1} \cap L_{2} & =L_{1}^{\prime}\left[x_{1}:=L_{1}^{\prime \prime}\right]_{I O} \cap L_{2}^{\prime}\left[x_{2}:=L_{2}^{\prime \prime}\right]_{I O} \\
& =\left(\left(L_{1}^{\prime} \cap L_{2}^{\prime}\right)\left[x:=L_{1}^{\prime \prime}\right]_{I O}\right)\left[x:=L_{2}^{\prime \prime}\right]_{I O} \\
& =\left(\left(L_{1}^{\prime} \cap L_{2}^{\prime}\right)\left[x:=L_{2}^{\prime \prime}\right]_{I O}\right)\left[x:=L_{1}^{\prime \prime}\right]_{I O}
\end{aligned}
$$

and $L_{1}^{\prime} \cap L_{2}^{\prime}$ is a $I O-M C L F$ by induction hypothesis, (the result is given in the case $L_{1}^{\prime}$ and $L_{2}^{\prime}$ are MCFLs) which implies $L_{1} \cap L_{2}$ belongs to IO-MCFL by construction.

- we show $L_{1} \cdot L_{2}$ belongs to IO-MCFL in a similar way:

$$
\begin{aligned}
L_{1} \cdot L_{2} & =L_{1}^{\prime}\left[x_{1}:=L_{1}^{\prime \prime}\right]_{I O} \cdot L_{2}^{\prime}\left[x_{2}:=L_{2}^{\prime \prime}\right]_{I O} \\
& =\left(\left(L_{1}^{\prime} \cdot L_{2}^{\prime}\right)\left[x:=L_{1}^{\prime \prime}\right]_{I O}\right)\left[x:=L_{2}^{\prime \prime}\right]_{I O} \\
& =\left(\left(L_{1}^{\prime} \cdot L_{2}^{\prime}\right)\left[x:=L_{2}^{\prime \prime}\right]_{I O}\right)\left[x:=L_{1}^{\prime \prime}\right]_{I O}
\end{aligned}
$$

and $L_{1}^{\prime} \cdot L_{2}^{\prime} \in I O-M C L F$ by induction hypothesis, (the result is given in the case $L_{1}^{\prime}$ and $L_{2}^{\prime}$ are MCFLs) which implies $L_{1} \cdot L_{2}$ is a IO-MCFL by construction.

- the closure by Kleene star is a generalization of the previous result.

Property 1. The family IO-MCFL is closed by intersection with a regular set.

Proof. Let us consider a IO-MCFL $L$ and a regular language $L$. We prove that $L \cap R$ belongs to IO-MCFL, by induction on $L$. If $L$ is a MCFL, the result is given by the fat that MCFLs form a AFL. Let us suppose $L=L_{1}\left[x:=L_{2}\right]_{I O}$. We consider $\mathbb{M}_{R}=(\mathscr{M}, \cdot, \epsilon)$ the syntactic monoid of $R$, where $\mathscr{M}$ is finite according to Myhill-Nerode. Then, there exists a homomorphism $\phi: \Sigma \mapsto \mathbb{M}_{R}$ and $\mathscr{N} \subseteq \mathbb{M}_{R}$ such that $R=\phi^{-1}(\mathscr{N})$. Given $m \in \mathscr{M}$ let us consider the homomorphism $\phi_{m}: \Sigma \cup\{x\} \mapsto \mathbb{M}_{R}$ defined by $\phi_{m}(x)=m$ and for every $a \in \Sigma, \phi_{m}(a)=\phi(a)$. We consider the regular set $R_{m}=\phi_{m}^{-1}(\mathscr{N})$. By induction hypothesis, $L_{1, m}=L_{1} \cap R_{m}$ is in IO-MCFL. The language $L_{2, m}=L_{2} \cap \phi^{-1}(\{m\})$ enjoys the same property, and by construction, $L_{m}=L_{1, m}\left[x:=L_{2, m}\right]_{I O}$ belongs to IO-MCFL. Finally, because $L \cap R=\bigcup_{m \in \mathscr{M}} L_{m}$, which is a finite union, and IO-MCFL is closed by union, we can conclude that $L \cap R$ is a IO-MCFL.

While all these properties can be proved in quite easily, the closure under inverse homomorphism does not seem as trivial. Actually, we can conjecture that such a property does not stand. 


\section{Conclusion}

\section{References}

[Bar84] Henk Barendregt. $\lambda$-calculus: its syntax and semantics. Elsevier Science Publishers Ltd., 1984.

[BS11] Pierre Bourreau and Sylvain Salvati. A Datalog recognizer for almost affine $\lambda$-CFGs. In Makoto Kanazawa, Andreàs Kornai, Marcus Kracht, and Hiroyuki Seki, editors, MOL, volume 6878 of Lecture Notes in Artificial Intelligence, pages 21-38. Springer, 2011.

[dG01] Philippe de Groote. Towards abstract categorial grammars. In Association for Computational Linguistics, 39th Annual Meeting and 10th Conference of the European Chapter, Proceedings of the Conference, pages 148-155, 2001.

[dGP04] Philippe de Groote and Sylvain Pogodalla. On the expressive power of abstract categorial grammars: Representing context-free formalisms. Journal of Logic, Language and Information, 13(4):421-438, 2004.

[Fis68a] Michael J. Fischer. Grammars with macro-like productions. In IEEE Conference Record of 9th Annual Symposium on Switching and Automata Theory, pages 131142. IEEE, 1968.

[Fis68b] Micheal J. Fischer. Grammars with macro-like productions. PhD thesis, Harvard University, 1968.

[JLT75] Aravind K. Joshi, Leon S. Levy, and Masako Takahashi. Tree adjunct grammars. J. Comput. Syst. Sci., 10(1):136-163, 1975.

[Jos85] Aravind K. Joshi. Tree-adjoining grammars: How much context-sensitivity is required to provide reasonable strucutral descriptions? Natural Language Parsing: Psychological, Computational and Theoretical Perspectives, pages 206-250, 1985.

[Kal10] Laura Kallmeyer. On mildly context-sensitive non-linear rewriting. Research on Language and Computation, 8(2):341-363, 2010.

[Kan06] Makoto Kanazawa. Abstract families of abstract categorial grammars. In Stanford University CSLI, editor, Proceedings of WoLLIC, 2006.

[Kan07] Makoto Kanazawa. Parsing and generation as Datalog queries. In Proceedings of the 45th Annual Meeting of the Association for Computational Linguistics, pages 176-183, Prague, 2007. Association for Computational Linguistics.

[Mus01] Reinhard Muskens. Lambda Grammars and the Syntax-Semantics Interface. In R. van Rooy and M. Stokhof, editors, Proceedings of the Thirteenth Amsterdam Colloquium, pages 150-155, Amsterdam, 2001.

[SMMK91] Hiroyuki Seki, Takashi Matsamura, Fujii Mamoru, and Tadao Kasami. On multiple context-free grammars. Theoretical Computer Science, 88(2):191-229, 1991.

[SU06] Morten Heine Sørensen and Pawel Urzyczyn. Lectures on the Curry-Howard isomorphism. Elsevier Science, 2006.

[Wei88] David Weir. Characterizing Mildly Context-Sensitive Grammar Formalisms. PhD thesis, University of Pennsylvania, 1988.

[Yos06] Ryo Yoshinaka. Linearization of affine abstract categorial grammars. In Proceedings of the 11th Conference on Formal Grammar, pages 185-199, Malaga, Spain, 2006. 